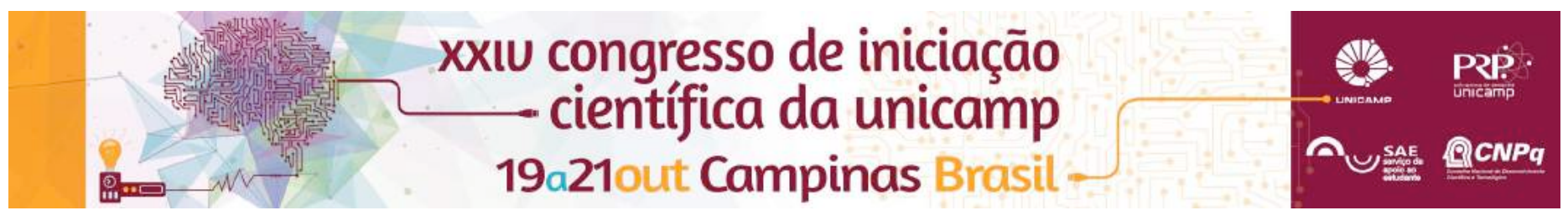

\title{
Collagen fiber analysis in melanoma: a new candidate to precise lesion border
}

\author{
Lucas A. Cavalcante*, Rodrigo A. Natal, Vitor B. Pelegati, Carlos L. Cesar, José Vassallo
}

\begin{abstract}
In melanoma, collagen has been described as a potential new prognostic biomarker. In this way, our purpose was to evaluate the quantity, uniformity and organization of peri- and intratumoral collagen, and to correlate these parameters with clinicopathological features. For this purpose, a total of 22 specimens of cutaneous melanoma were submitted to second-harmonic generation microscopy and image evaluation. Our results suggest that the collagen fiber parameters, mainly organization, are potential candidates to determine with higher precision the border between neoplasia and nonneoplastic dermis.
\end{abstract}

\section{Key words: Melanoma; Second-harmonic generation image; Collagen; Diagnosis}

\section{Introduction}

Non linear optics, including two-photon excited fluorescence (TPEF) and second-harmonic generation (SHG), has provided a breakthrough in collagen research in many diseases. In melanoma, collagen has been described as a potential new prognostic biomarker, since it can influence tumor growth, invasion and metastasis. In this perspective, our purpose was to evaluate the quantity, uniformity and organization of peri- and intratumoral collagen, and to correlate these parameters with clinicopathological features.

\section{Materials and Methods}

22 samples of paraffin embedded human melanoma were sectioned $(4 \mu \mathrm{m})$ and stained with hematoxylin and eosin. Slides were analyzed in an Inverted Zeiss LSM 780-NLO confocal system (Carl Zeiss AG, Germany). Three SHG images from intratumoral (close to melanoma cells) and three from peritumoral (skin distant from melanoma) collagen fibers were captured with an excitation wavelength of $800 \mathrm{~nm}$, a pulse length of approximately $100 \mathrm{fs}$, and emission filter of SP405 nm (Semrock, Rochester, NY, USA) (Figure 1). Images were analyzed in 3 randomly choosen regions for each image, using the ImageJ software $(\mathrm{NIH}$, available at http://rsb.info.nih.gov/ij). Collagen fiber quantity, uniformity and organization of peri- and intratumoral areas were compared using t-test, and the sensitivity and specificity for collagen parameters to distinguish peri- and intratumoral areas were assessed with ROC-curve, using RStudio (CRAN, available at https://www.rstudio.com). Significance was set at $p<0.05$.

\section{Results and Discussion}

The median age of patients was 58 years $(31-81$ years). The tumor was Clark stage I in $09(40.9 \%)$ patients, II in $03(13.6 \%)$, III in $06(27.3 \%)$, IV in $02(9,1 \%)$ and $V$ in $02(9,1 \%)$. The median Breslow was $1,07 \mathrm{~mm}(0.0-6.3$ $\mathrm{mm})$. Quantitative analyses of the SHG images demonstrated that intratumoral collagen fibers showed high values of collagen parameters (Table 1 ) in relation to peritumoral collagen fibers. ROC curve was constructed for all parameters to verify the potential of differentiation between peri- and intratumoral collagen: quantity showed $52, . \%$ sensitivity and $90.5 \%$ specificity; uniformity, $61.9 \%$ and $85.7 \%$, and organization $42.9 \%$ and $100 \%$.

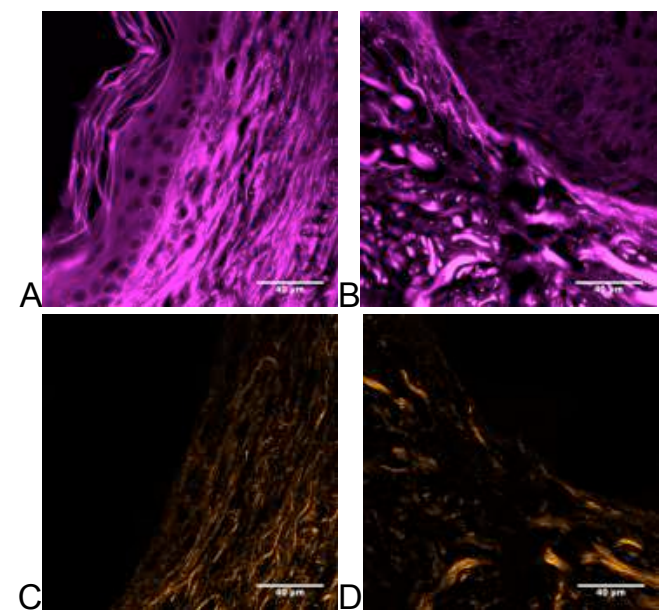

Figure 1: Representation of autofluorescence tissue sections (A and $B$ ) and SHG images (C and $D)$, in peri- $(A$ and $C)$ and intratumoral ( $B$ and D) areas.

Table 1. Results for peri- and intratumoral collagen fiber parameters.

\begin{tabular}{llll}
\multicolumn{4}{l}{ Table 1. Results for peri- and intratumoral collagen fiber parameters. } \\
\hline & Peritumoral & Intratumoral & $p$-value \\
\hline Quantity & $14.26 \pm 0.62$ & $14.76 \pm 0.63$ & $<0.001$ \\
Uniformity & $9.41 \pm 0.74$ & $10.05 \pm 0.77$ & $<0.001$ \\
Organization & $0.08 \pm 0.04$ & $0.14 \pm 0.06$ & $<0.001$ \\
\hline
\end{tabular}

\section{Conclusions}

Our results suggest that the collagen fiber parameters, mainly organization, are candidates to determine lesion borders with higher precision.

\section{Acknowledgement}

We thank the National Institute of Science and Technology on Photonics Applied to Cell Biology (INFABiC) at the State University of Campinas for access to equipment and assistance; INFABiC is co-funded by Fundação de Amparo à Pesquisa do Estado de São Paulo (FAPESP) (08/57906-3) and Conselho Nacional de Desenvolvimento Científico e Tecnológico (CNPq) (573913/2008-0).

Hompland T, Erikson A, Lindgreen M, Lindmo T, de Lange Davies C. "Second-harmonic generation in collagen as a potential cancer diagnostic parameter". J Biomed Opt. 13, 054050 (2008).

Pei-Chun W, Tsung-Yuan H, Zen-Uong T, Tzu-Ming L. "In vivo Quantification of the structural changes of collagens in a melanoma microenvironment with second and third harmonic Generation Microscopy" Sci Rep. 9, 8879 (2015). 